\title{
Improved Performance Robustness of DSP-Enabled Flexible ROADMs Free from Optical Filters and O-E-O Conversions
}

\author{
Wei Jin, Chong F. Zhang, Xiao Duan, Mohammad R. Kadhum, Yi X. Dong, Roger P. Giddings,
} Ning Jiang, Kun Qiu and J. M. Tang

networking (SDN) functionalities [3, 4] to dynamically provide

\begin{abstract}
Utilizing Hilbert-pair-based digital filtering, intensity modulation and passive optical coupling, DSP-enabled flexible ROADMs are reported, which are free from both optical filters and O-E-O conversions and also offer excellent flexibility, colorlessness, gridlessness, contentionlessness, adaptability and transparency to physical-layer network characteristics. In this paper, the ROADM performance robustness against variations in numerous network design aspects is, for the first time, extensively explored in IMDD-based optical network nodes. Numerical results show that DSPs not only enable the ROADMs to dynamically and flexibly perform add/drop operations at wavelength, subwavelength and spectrally overlapped orthogonal sub-bands levels, but also considerably improve the ROADM performance robustness against variations in modulation formats, transmission system characteristics/impairments, as well as terminal equipment configurations.
\end{abstract}

Index Terms-Digital filtering, digital signal processing (DSP), intensity modulation, reconfigurable optical add/drop multiplexer (ROADM), performance robustness.

\section{INTRODUCTION}

$\mathrm{D}$

riven by the ever-increasing traffic growth, cloud access networks $[1,2]$ have been proposed to seamlessly integrate traditional optical access networks, metropolitan optical networks and mobile front-haul/back-haul networks. From practical application point of view, it is greatly advantageous if the cloud access networks have not only strong adaptability to highly dynamic traffic with arbitrary bandwidth granularity, but also desirable performance capability of simultaneously accommodating a wide diversity of key network operation features including, for example, various signal modulation formats, different signal detection schemes, flexible wavelength division multiplexing (WDM) grids, diversified network topologies and various multiple access techniques. In addition, it is also preferable to equip the cloud access networks with a diversity of software-defined

W. Jin, C. F. Zhang, N. Jiang and K. Qiu are with Key Laboratory of Optical Fiber Sensing and Communications, University of Electronic Science and Technology of China, Chengdu, 611731, China. (weijinmail@ foxmail.com).

W. Jin, X. Duan, M.R. Kadhum, Y. X. Dong, R. P. Giddings and J. M. Tang are also with the School of Electronic Engineering, Bangor University, Bangor, LL57 1UT, UK. (j.tang@bangor.ac.uk). fast on-demand connections/services at wavelength, subwavelength and sub-band levels.

As one of the most important networking devices offering fast connectivity between an expanded number of individual optical networks, reconfigurable optical add/drop multiplexers (ROADMs) [5,6] with advanced architectures and flexible functionalities are expected to play a vital role in ensuring the achievement of all the aforementioned networking features required by cloud access networks, whilst still allowing a technical strategy of significantly reducing both the CapEx and the OpEx.

Recently, a number of new ROADM architectures have been reported with advanced functionalities in terms of colorlessness, directionlessness, contentionlessness and WDM-gridlessness [7-14]. However, those ROADMs are constructed using hardwired switching elements, this significantly limits the ROADM's upgradability, flexibility and adaptability at subwavelength and sub-band levels [15]. On the other hand, given the fact that the cloud access networks are very cost-sensitive and dynamic, optical-electrical-optical (O-E-O)-free, SDNcontrollable ROADM configurations are also highly desirable.

Utilizing intensity modulations, passive optical coupling and digital filtering, SDN-controllable, colorless, contentionless, WDM-gridless, O-E-O conversion-free ROADMs [16] have been reported to offer DSP-enabled flexible and tunable add/drop operations at wavelength, sub-wavelength and spectral overlapped orthogonal sub-bands levels in simple intensity modulation and direct detection (IMDD)-based optical network nodes. However, in such ROADMs, costly narrow optical tunable filters are employed to perform the ROADM add/drop operations. To overcome this drawback and further enhance the ROADM practicability, DSP-enabled O-E-O conversion- and optical filter-free ROADMs [17] have been proposed without compromising above-mentioned salient ROADM features. In addition, to rigorously verify the ROADM design and further evaluate its operation impacts on physical-layer network performance, very recently, experimental demonstrations of optical filter- and O-E-O conversion-free ROADM drop operations at spectrally overlapped orthogonal sub-band level have been reported utilizing commercially-available, low-cost electrical/optical 


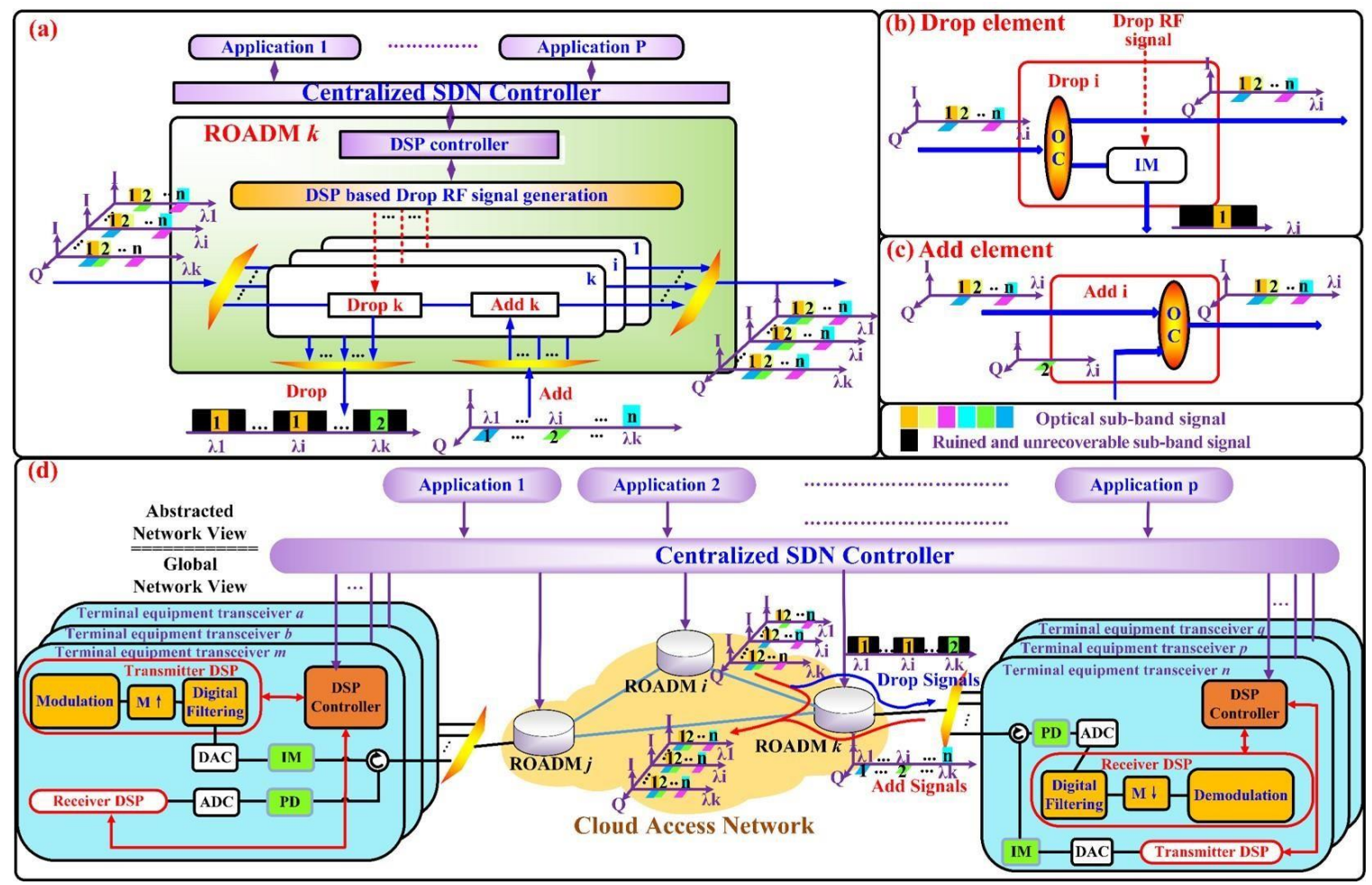

Fig. 1. (a) Schematic diagram of a DSP-enabled ROADM without optical filters and O-E-O conversion; (b) drop element; (c) add element; (d) representative entire network architecture incorporating the proposed ROADMs. The orthogonal digital filtering-associated DSP functions implemented in corresponding TETs are also revealed in Fig. 1(d). IM: intensity modulator, OC: optical coupler.

components [18]. Experimental results have shown similar bit error rate (BER) performances for orthogonal sub-band signals before and after the drop operation [18]. This verifies the theoretical predictions [17].

Our previous works reported in [16-18] have, however, mainly been focused on the ROADM performances using optical orthogonal frequency division multiplexing (OFDM) signals only. From practical application point of view, it is also necessary to investigate the proposed ROADM performance robustness against variations in numerous key network design aspects including signal modulation formats, embedded DSP algorithms, transmission system characteristics/impairments, as well as terminal equipment configurations under various application scenarios. Addressing such challenges is the main scopes of this paper. In addition, special attention is also given to explore the feasibility of using DSP to further improve the ROADM performance robustness.

\section{ROADM Operating PRINCIPLE For QAM-ENCODED OPTICAL SIGNALS}

The schematic diagram of a centralized SDN controllercontrollable ROADM architecture is shown in Fig. 1(a). An intensity modulator (IM)-based drop element driven by a SDN controlled radio frequency (RF) signal is illustrated in Fig. 1(b) and an optical coupler (OC)-based add element is also shown in Fig. 1(c). In addition, a representative entire network architecture incorporating the proposed ROADMs is also illustrated in Fig. 1(d), where the orthogonal digital filteringassociated DSP functions implemented in corresponding terminal equipment transceivers (TETs) are also revealed.
Detailed descriptions of the ROADM operating principle can be found in [17]. In the ROADM, the input and output optical signal of a specific wavelength consists of multiple subwavelength bands each at a different RF frequency. Each individual sub-wavelength band can have either two digital filtering-enabled spectrally overlapped orthogonal sub-bands (In-phase "I" and Quadrature-phase "Q" sub-bands) or a single sub-band (I or Q sub-band). To drop a targeted sub-band of a specific wavelength in the drop element, the optical signal passes through an IM driven by a SDN-controllable drop RF signal, as shown in Fig. 1(b). At the IM output, the dropped subband with a reversed signal spectrum is shifted to the baseband spectral region, and all other sub-bands of the same wavelength are ruined and unrecoverable. On the other hand, very similar to the procedure reported in [19], in the add element, the add operation at a specific wavelength is performed by passive optical coupling in an optical coupler, as illustrated in Fig.1(c).

An optical signal at an optical carrier frequency, $f_{0}$, containing $\mathrm{N}$ quadrature amplitude modulation

(QAM)-encoded signals, can be expressed as:

$$
\operatorname{Sopt-QAM}(t)=\sum N i=-01 y_{i}(t) e_{j 2 \pi f 0 t}
$$

where $y_{i}(t)$ is the $\mathrm{i}$-th $\mathrm{QAM}$ signal, whose $\mathrm{I}_{\mathrm{i}}$ and/or $\mathrm{Q}_{\mathrm{i}}$ components are firstly up-sampled and subsequently digitally filtered by a pair of digital filters in a TET. Considering the fact that the $\mathrm{I}_{\mathrm{i}}$ and $\mathrm{Q}_{\mathrm{i}}$ components of the QAM signal occupy two individual sub-bands, to drop the i-th QAM signal, $y_{i}(t)$, two parallel IMs are utilized with their SDN controlled drop RF signals written as: 


$$
\begin{array}{ccc}
k_{e j 2 \pi f i t} & \left.\frac{1+1}{1+k}\right) & \operatorname{Re}( \\
\left\{\begin{array}{c}
\operatorname{Re}() \\
i^{w}-\operatorname{Drop}(t)=\frac{1+k e^{j\left(2 \pi f_{i 2} t+\frac{\pi}{2}\right)}}{1+k}
\end{array}\right. & \\
& &
\end{array}
$$

where $k(0<k<1)$ is the parameter introduced to ensure that the drop RF signal amplitude varies within a dynamic range from 0 to 1. A large $k$ parameter can improve the ROADM drop operation performance [16]. $f_{i 1}$ and $f_{i 2}$ are the central RF frequencies of the i-th digital filter pair employed by the i-th QAM signal. After the drop operation in the TET end, these two dropped optical signals can be directly detected and then downsampled. The $\mathrm{I}_{\mathrm{i}}$ and $\mathrm{Q}_{\mathrm{i}}$ components of the received i-th QAM signal after down-sampling can be expressed as:

$$
\begin{aligned}
& S_{\text {Recieved-i-QAM }}=I_{i}(n T)-j Q_{i}(n T) \\
& =(-1) n\left[\gamma_{i 1} I i(n T) e_{j \varphi i 1}+j \gamma_{i 2} Q_{i}(n T) e_{j \varphi i 2}\right]
\end{aligned}
$$

where $\varphi_{i 1}$ and $\varphi_{i 2}$ are the phase differences between the received $\left(\mathcal{I}_{i}, Q_{i}\right)$ and transmitted $\left(I_{i}, Q_{i}\right)$ components of the i-th QAM signal. $\gamma_{i 1}$ and $\gamma_{i 2}$ represent the drop operation-induced amplitude variation for the $I_{i}$ and $Q_{i}$ component, respectively. $(-1)^{n}$ is introduced to account for the reversed signal spectrum caused by the combined effects of digital filtering and drop operation [17].

It is also worth mentioning the following five aspects: i) The ROADMs can also perform the add/drop operation for a QAM signal with its I and Q components occupying one or two subwavelength bands; ii) The ROADM add operation of a QAM signals is identical to those explicitly reported in [17]; iii) Via selecting two free digital filters and/or shifting the central frequencies of these two digital filters to free spectral regions, the digital filtered QAM signal can locate at free spectral regions to enable the contention-free add operation; iv) According to the network traffic status, all the RF signals required to perform the add/drop operations could alternatively be dynamically generated in the digital domain. This has the potential to significantly reduce the number of ROADM-associated RF components, thus leading to considerable improvements in both cost-effectiveness and power consumption efficiency. RF amplifiers may still be required to achieve sufficient IM drive levels, however power efficient switching amplifiers are now emerging for $\mathrm{GHz}$ frequencies; and v) It is only necessary for a single ROADM to have RF synthesizers corresponding to the maximum number of dropped sub-bands within the ROADM which can be a subset of the total number of sub-bands in the system. Given the fact that the harmonic relationship between the required RF frequencies, frequency dividers can be exploited to reduce power consumption and reduce device cost. Due to the above-mentioned ROADM features, the ROADMs are thus adaptive, flexible, transparent and contention-free.

It is also envisaged that an application-specific integrated circuit (ASIC)-based, highly-integrated ROADM should meet

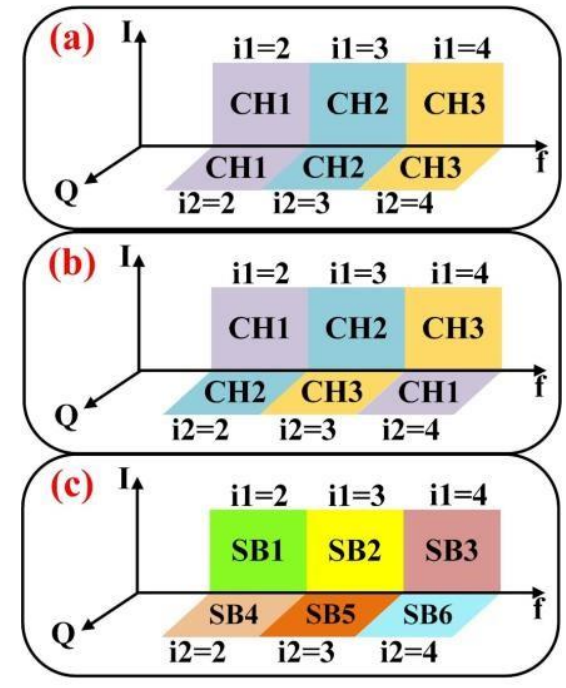

Fig. 2. Spectral locations of digital filtered QAM signals and OFDM subbands in various sub-wavelength band spectral regions; (a) three OSOSW QAM signals; (b) three OSTSW QAM signals; (c) six OFDM sub-bands. $\mathrm{CH}$ : channel, SB: sub-band, I: in-phase, Q: quadrature-phase.

TABLE I

BITRATE OF EACH OFDM AND QAM SIGNAL (GB/S)

\begin{tabular}{|c|c|c|c|c|}
\hline Digital Filter Length (L) & $\mathrm{L}=16$ & $\mathrm{~L}=32$ & $\mathrm{~L}=64$ & $\mathrm{~L}=128$ \\
\hline $\begin{array}{l}\text { OSOSW LMS Taps Number case } \\
\text { LMS Step Size }\end{array}$ & $\int_{3}^{2510}$ & $\begin{array}{ll}4310 \\
3\end{array}$ & $\begin{array}{ll}7310 \\
4\end{array}$ & $\begin{array}{l}135 \\
10-4\end{array}$ \\
\hline $\begin{array}{l}\text { OSTSW LMS Taps Number case } \\
\text { LMS Step Size }\end{array}$ & $\begin{array}{l}1 \\
1\end{array}$ & ${ }_{3}^{43} 10$ & ${ }_{3}^{7310}$ & $\begin{array}{l}135 \\
10-4\end{array}$ \\
\hline
\end{tabular}

\begin{tabular}{cccccc}
\hline \hline & 4QAM & 8QAM & 16QAM & 32QAM & OFDM \\
\hline $\begin{array}{c}\text { Bitrate per } \\
\text { Channel } \\
\text { Total } \\
\text { Bitrates }\end{array}$ & 3.71 & 5.56 & 7.42 & 9.28 & 2.91 \\
\hline \hline
\end{tabular}

TABLE II

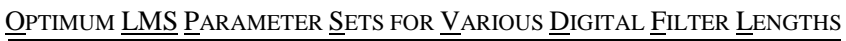

the c2011 GPON requirements in the EC Code of Conduct Energy Consumption of Broadband Equipment. The requirements allow up to $7.7 \mathrm{~W}$ for core home gateway functions in the active state and $4 \mathrm{~W}$ in the idle state. This suggests that such a ROADM may be capable of reducing the power consumption by a factor of 10 compared to commerciallyavailable conventional ROADMs.

\section{ROADM PERFORMANCE ROBUSTNESS}

Throughout this paper, use is made of the Hilbert-pair approach to construct the required digital filters [19-21]. As detailed descriptions of the digital filtering of OFDM signals are explicitly presented in [17], here special attention is, therefore, focused on the digital filtering of QAM signals only. In a TET, the transmitted i-th QAM signal is firstly up-sampled and then digitally filtered to generate $y_{i}(t)$ with its form expressed as:

$y_{i}(t)=I_{i-u p s a m p l e d}(t) \otimes h_{i I}(t)-Q_{i-u p s a m p l e d}(t) \otimes h_{i Q}$ where I-upsampled $(t)$ and $Q_{i-u p s a m p l e d}(t)$ are the I and Q 
components of the up-sampled i-th QAM signal. $h_{i}{ }^{I}(t)$ and $h_{i}{ }^{Q}$ are the impulse responses of the i-th digital filter pair employed by the QAM signal. For a specific wavelength transmitting $N$ independent QAM signals, the central RF frequencies of the

i-th digital filter pair, $\left\{f_{i 1}, f_{i 2}\right\}$, are given by [19],

$$
\begin{gathered}
\left\{f_{i 1}=(2 \times i 1-1) \frac{f D A C / A D C}{4 N} \quad i 1=1,2, \ldots, N\right. \\
f_{i 2}=(2 \times i 2-1)^{\frac{f D A C / A D C}{4 N}} \quad i 2=1,2, \ldots, N
\end{gathered}
$$

where $f_{D A C / A D C}$ is the sampling speed of the digital-toanalogue/analogue-to-digital converter (DAC/ADC).

When $f_{i 1}$ is equal to $f_{i 2}$, the two digital filters are orthogonal and spectrally overlapped. This results in the filtered QAM signal to occupy a whole sub-wavelength spectral region. Whilst when $f_{i 1} \neq f_{i 2}$, the I and Q components of a digital filtered QAM signal occupy two different sub-wavelength bands.

Three digital filter pairs are adopted in this paper, their digital filter lengths of $\mathrm{L}=64$ and excess of bandwidths of $\alpha=$ 0 are taken, and their central frequencies satisfy Eq. 5 with \{ $i 1=2,3,4 ; i 2=2,3,4\}$. The up-sampling factor, $\mathrm{M}$, is fixed at 8. The sampling speed of a DAC/ADC with 8-bit resolution is $15 \mathrm{GS} / \mathrm{s}$. To highlight the ROADM performance robustness, ideal intensity modulators are utilized to perform the drop operation. To directly detect a dropped optical signal, an APD is employed with a quantum efficiency of 0.8 and a power sensitivity of $-26 \mathrm{dBm}$.

To investigate the ROADM performance robustness to signal modulation format variations, six OFDM signals and three QAM signals ranging from 4-QAM, 8-QAM, 16-QAM to 32-QAM are adopted. Each QAM signal sequence contains $2 \times 10^{4}$ symbols with the first 200 symbols acting as a training sequence. In this paper, two cases are considered: 1) onesignal-occupying-one-sub-wavelength-band (OSOSW), i.e., a QAM signal with its I and Q component central frequencies satisfying $f_{i 1}=f_{i 2}$; and 2) one-signal-occupying-two-subwavelength-bands (OSTSW), i.e., a QAM signal with its I and $\mathrm{Q}$ component central frequencies satisfying $f_{i 1} \neq f_{i 2}$. For each QAM modulation format, the above-mentioned parameters result in three optical OSOSW QAM signals and three optical OSTSW QAM signals, their I and Q component spectral locations are illustrated in Figs. 2(a) and 2(b), respectively. Using parameters adopted in [17] to generate adaptively bitand power-loaded OFDM signals, six OFDM sub-bands are also produced with their sub-band spectral locations shown in Fig. 2(c). The optical power of each optical QAM and OFDM signal is fixed at $-2 \mathrm{dBm}$ and their bit rates are summarized in Table I.

To recover a received QAM signal after down-sampling at a TET end, a standard least mean square algorithm (LMS) [22] is utilized following training sequence assisted-I/Q signal power adjustments. For both the OSOSW and OSTSW cases, the numerically identified optimum LMS parameter sets for
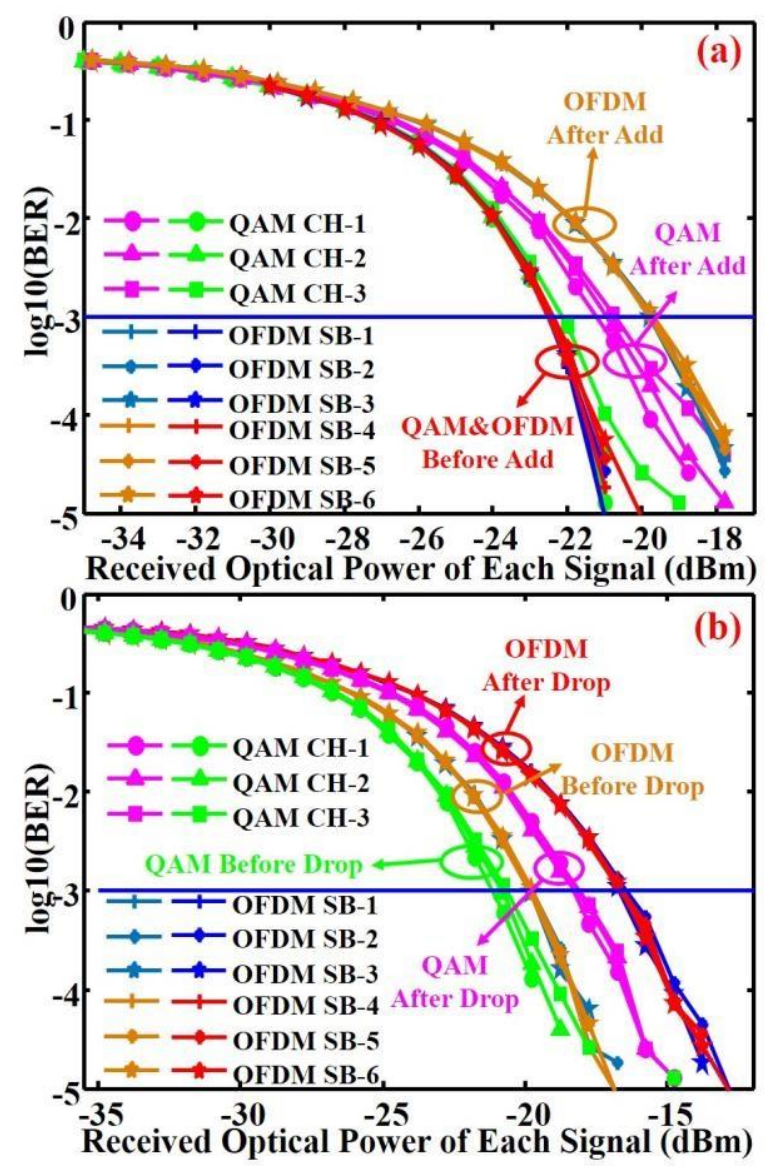

Fig. 3. BER performances of OFDM and OSOSW 16-QAM signals; (a) before and after add operation; (b) before and after drop operation. $\mathrm{CH}$ : channel, SB: sub-band.

different digital filter lengths are listed in Table II, which give rise to the lowest LMS DSP complexity but without compromising the signal recovery performance. It is also worth mentioning that the training sequences are used to not only compensate for the ROADM operation-induced I/Q signal amplitude/phase variations, but also accelerate the convergence of the LMS digital equalizers.

\section{A. Add/drop performance robustness to signal modulation formats}

Utilizing the parameters explicitly stated above, six OFDM sub-bands and three OSOSW 16-QAM signals are utilized to perform the add operation. The BER performances of these signals before and after performing the add operation are presented in Fig. 3(a), which shows that, before and after the add operation, three OSOSW QAM signals (six OFDM signals) have identical BER developing curves. This indicates that the ROADM add operation is sub-wavelength (sub-band) spectral location-independent for a given signal modulation format. For the OSOSW QAM signals, the add operation-induced power penalties (APPs) at BERs of $1.0 \times 10^{-3}$ of approximately $1.5 \mathrm{~dB}$ are mainly caused by the coexistence of the power leakage between different digital filters and a reduction in effective 
optical signal to noise ratio (OSNR). In addition, in comparison with the QAM signals, an extra $1 \mathrm{~dB}$ power penalty for the OFDM signals occurs mainly because of much lower signal
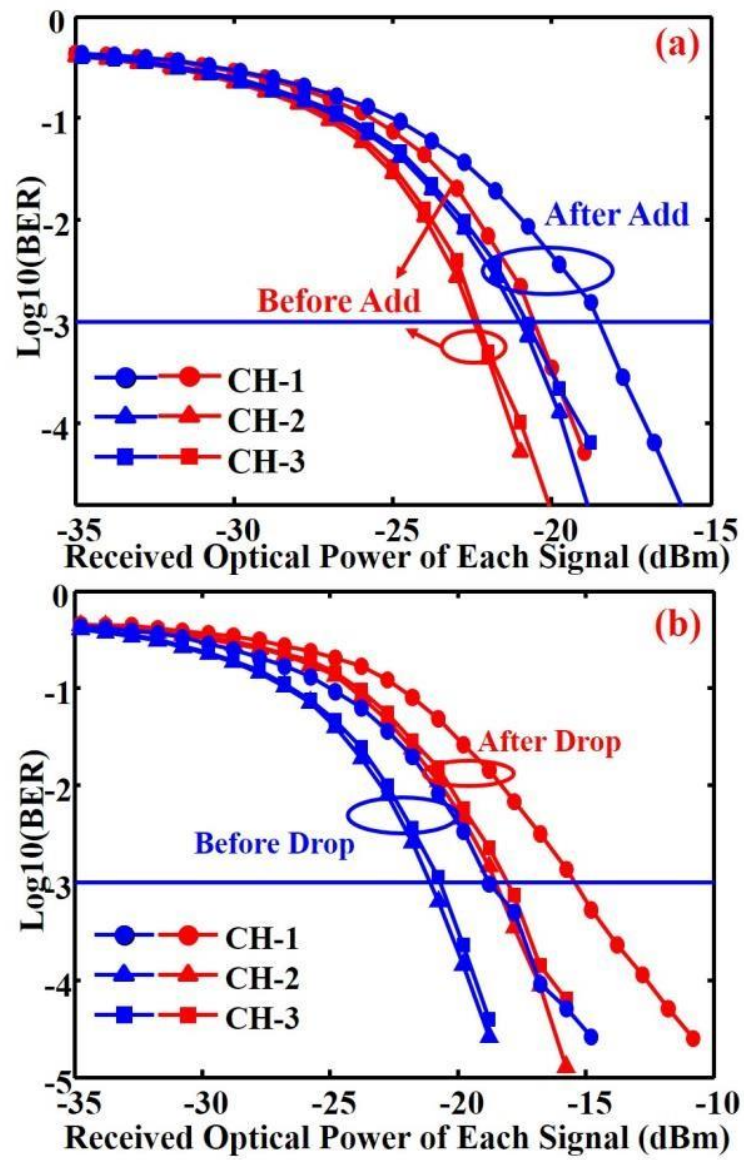

Fig. 4. BER performances of OSTSW 16-QAM signals; (a) before and after add operation; (b) before and after drop operation. $\mathrm{CH}$ : channel.

extinction ratios associated with these OFDM signals, which results in further reductions in effective OSNR after the add operation.

To perform the drop operation, digital filter lengths of $L=64$ and two optical signals of the same wavelength are adopted: one signal consisting of three OSOSW 16-QAM signals and the other consisting of six OFDM sub-bands. Making use of IMs with the $k$ parameter of 0.99 , the drop operation is performed for each QAM signal and OFDM sub-band. The resulting BER performances of these OFDM and QAM signals before and after the drop operation are shown in Fig. 3(b). It can be seen in Fig. 3(b) that very similar BER performances occur for both OSOSW QAM signals and OFDM signals before and after the drop operation, indicating sub-wavelength and sub-band locations-independent ROADM drop operation for a specific signal modulation format. It is also very interesting to note that, the identical drop operation-induced power penalties (DPPs) of about 3dB are observed for both OSOSW QAM and OFDM signals because of the ROADM drop operation-induced nonlinearities [18].

To investigate the impact of signal spectral location on the ROADM add performance, three OSTSW 16-QAM signals with their I and $\mathrm{Q}$ components located in different sub- wavelength regions illustrated in Fig. 2(b) are taken to perform the add operation. Based on the same digital filter length, the calculated BER performances of these OSTSW QAM signals before and after the add operation are presented in Fig. 4(a). Comparing Fig. 4(a) with Fig. 3(a), the receiver sensitivity degradations of QAM channel $1(\mathrm{CH}-1)$ at a BER of $1.0 \times 10^{-3}$ are mainly caused by the I/Q imbalanced digital filteringinduced degradations in signal recovery efficiency. On the other hand, the add operation gives rise to very similar power penalties for both OSOSW and OSTSW QAM signals, which are independent of their central RF frequencies, as expected from Fig. 3(a).

The impact of signal spectral location on the ROADM drop performance is explored in Fig. 4(b), where an optical signal consisting of three OSTSW 16-QAM signals with their I/Q component frequency locations presented in Fig. 2(b) is adopted and all other simulation parameters are identical to those adopted in Fig. 3(b). The BER performances of these OSTSW QAM signals before and after the drop operation are shown in Fig. 4(b). Comparing Fig. 4(b) with Fig. 3(b), the very similar drop operation-induced power penalties are observed for both OSOSW and OSTSW QAM signals.

Based on the simulation parameters identical to those adopted in Fig. 3 and Fig. 4, the ROADM performance robustness to signal modulation format variations is shown in Fig. 5, in which the receiver sensitivity, APP and DPP are plotted for different modulation formats. As expected, it is shown in Fig. 5 (c) and Fig. 5 (d) that, for both the OSOSW and OSTSW QAM signals, increasing the QAM modulation format level from 4-QAM to $32-\mathrm{QAM}$ just alters the APP (DPP) by $3 \mathrm{~dB}(2 \mathrm{~dB})$. This indicates the excellent ROADM performance robustness to modulation format variations. Such robustness can be further improved when digital filter lengths is longer than 64, as shown in Fig. 6. It is also shown in Fig. 5(d) that the OSTSW QAM signal with a large frequency interval between its I and Q component spectral locations suffers relative large APP and DPP, however, when narrowing such frequency interval, the BER performance of the OSTSW QAM signal is improved, and leads to BER curves similar to the OSOSW QAM signals.

\section{B. Add/drop performance robustness to digital filter length variations}

A long filter length increases the digital filter DSP complexity. By utilizing the simulation conditions similar to those adopted in computing Figs. 3 and 4, the ROADM add/drop performance robustness to digital filter length variations is investigated and the results are presented in Fig. 6. It is clear that a long digital filter length improves the ROADM add/drop performances, which agrees with numerically simulated results reported in [17]. From Fig. 6(a) and Fig. 6(c), it can be seen that, compared 

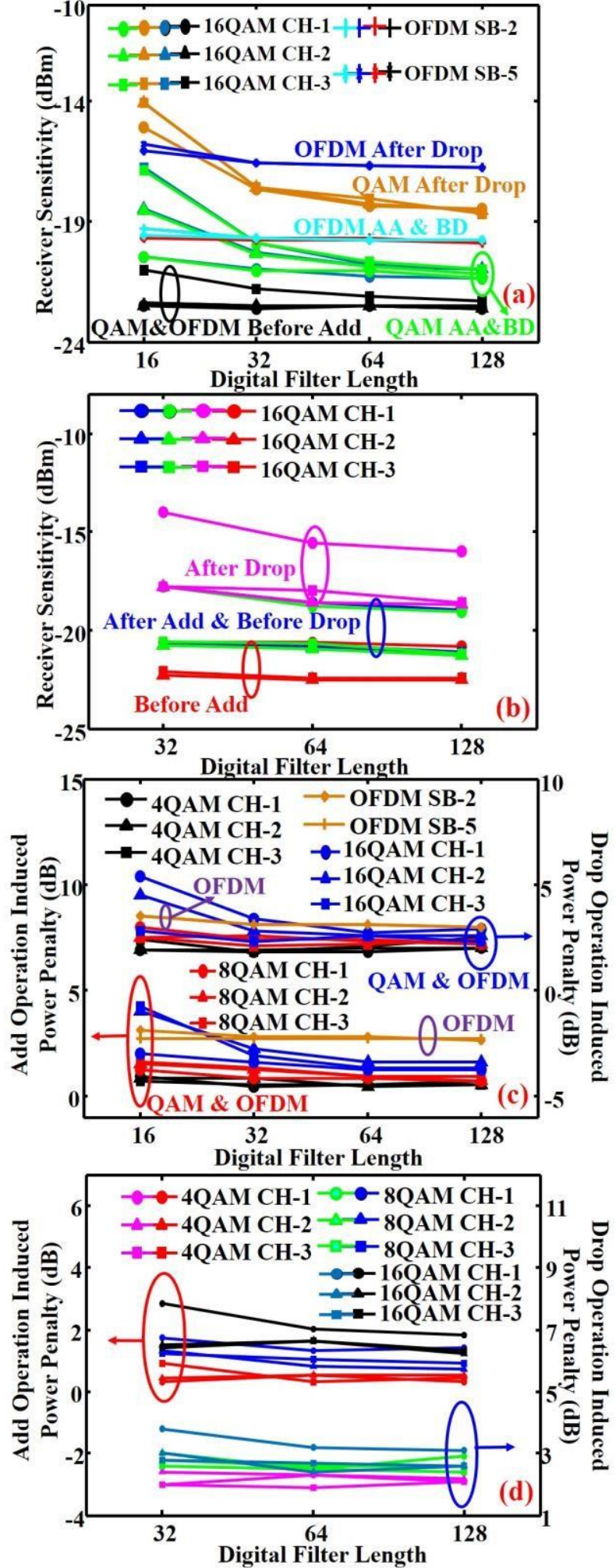

Fig. 5. ROADM add/drop performance robustness to modulation format variations; (a) receiver sensitivity variations for OSOSW QAM and OFDM signals; (b) receiver sensitivity variations for OSTSW QAM signals; (c) APP and DPP variations for OSOSW QAM and OFDM signals; (d) APP and DPP variations for OSTSW QAM signals. $\mathrm{CH}$ : channel, SB: sub-band, AA: after add, BD: before drop.
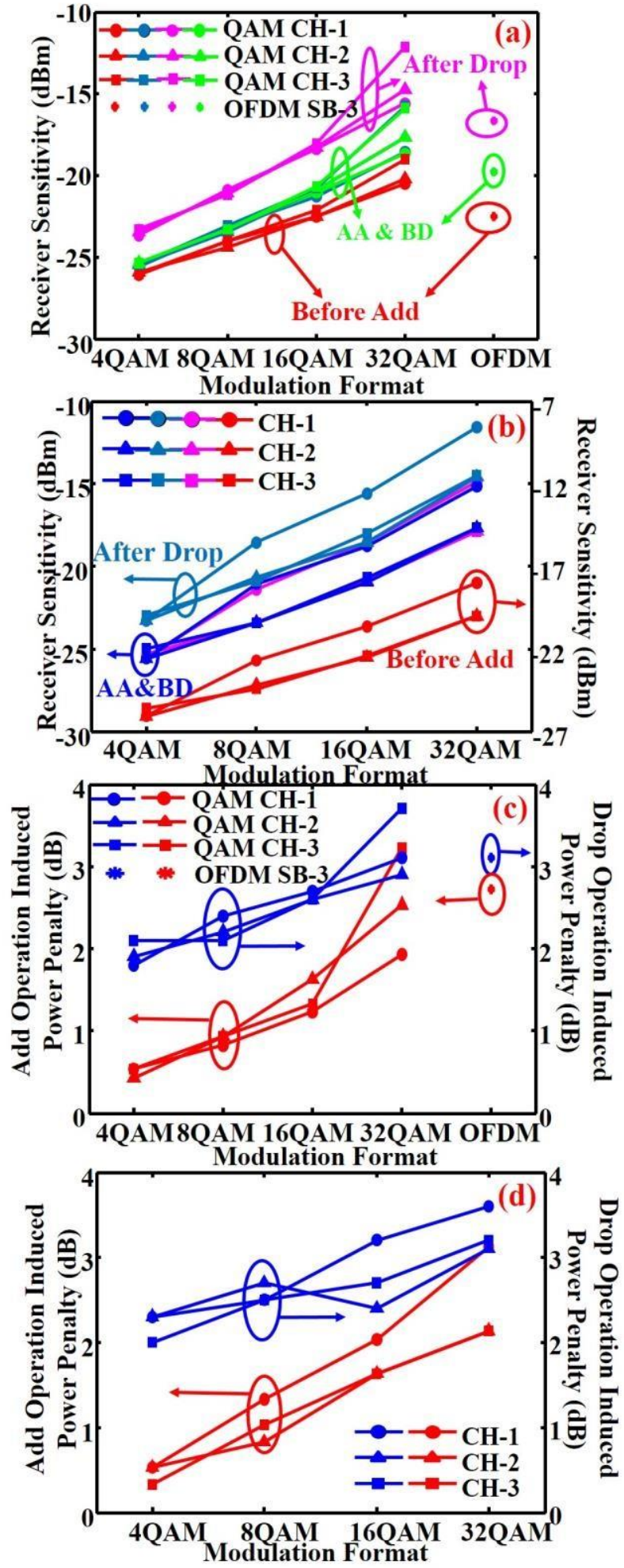

Fig. 6. ROADM add/drop performance robustness to digital filter length variations; (a) receiver sensitivity variations for OSOSW 16-QAM signals and OFDM signals; (b) receiver sensitivity variations for OSTSW 16-QAM signals; (c) APP and DPP variations for different OSOSW-QAM and OFDM modulation formats; (d) APP and DPP variations for different OSTSW QAM modulation formats. $\mathrm{CH}$ : channel, SB: sub-band, AA: after add, BD: before drop.

with QAM signals, OFDM signals slightly enhance the ROADM performance robustness due to the adoption of 
adaptive bit- and power-loading DSP algorithms. As expected, Figs. 6(c) and 6(d) also show that a relative low-order QAM modulation format enhances the ROADM performance robustness to digital filter length variations. The employment of digital filter length larger than

32 gives rise to APP and DPP variations of $\angle 3 \mathrm{~dB}$ for the OSOSW/OSTSW QAM and OFDM signals.

\section{Drop performance robustness to terminal equipment configurations}

To explore the ROADM drop performance robustness to various terminal equipment configurations, four different TET architecture cases outlined in Table III are considered: I) only TABLE III

EMPLOYMENTS OF OPTICAL/ELECTRICAL/DIGITAL FILTERS AT TET ENDS

\begin{tabular}{cccc}
\hline & $\begin{array}{c}\text { Baseband } \\
\text { Digital Filter }\end{array}$ & $\begin{array}{c}\text { Optical } \\
\text { Filter }\end{array}$ & $\begin{array}{c}\text { Electrical } \\
\text { Baseband Filter }\end{array}$ \\
\hline Case I & $\sqrt{ }$ & $\times$ & $\times$ \\
Case II & $\sqrt{ }$ & $\sqrt{ }$ & $\times$ \\
Case III & $\times$ & $\times$ & $\sqrt{ }$ \\
Case IV & $\times$ & $\sqrt{ }$ \\
\hline Filter & \multirow{2}{*}{$f_{D A C / A D C /(2 \mathrm{M})}$} & $f_{D A C / A D C}$ & $f_{D A C / A D C /(2 \mathrm{M})}$ \\
Bandwidth & &
\end{tabular}
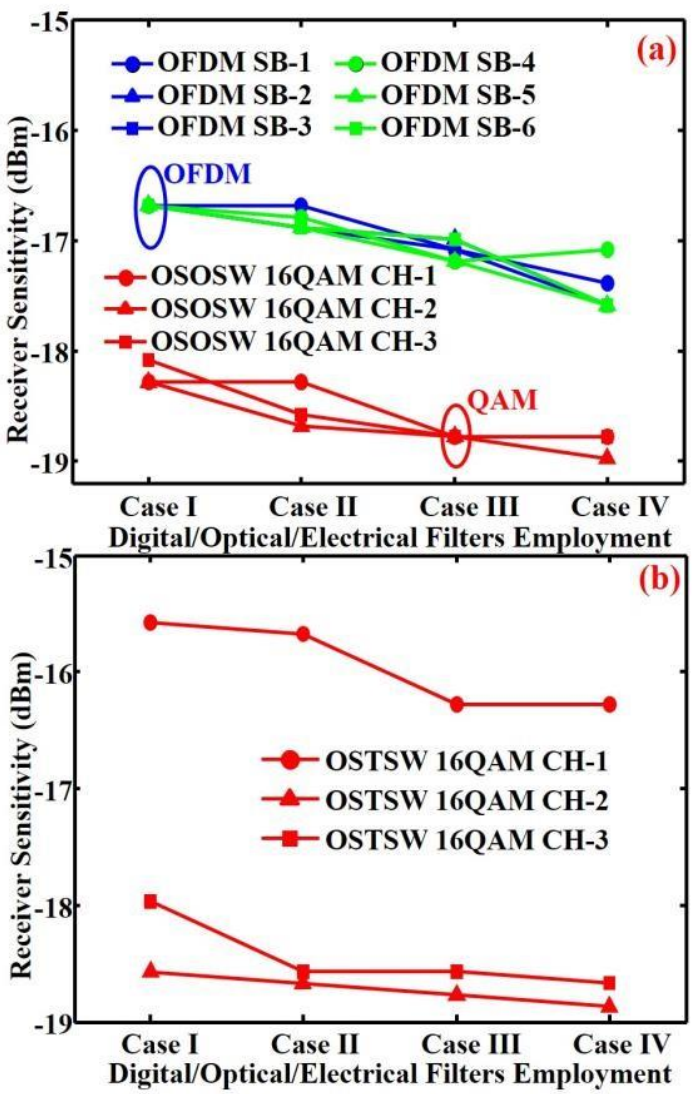

Fig. 7. ROADM drop performance robustness to TET configuration variations; (a) OSOSW 16-QAM and OFDM signals; (b) OSTSW 16QAM signals. $\mathrm{CH}$ : channel, $\mathrm{SB}$ : sub-band.

digital filtering is adopted without either electrical or optical filters; II) an optical filter with its bandwidth equal to
$f_{D A C / A D C}$ is used directly before the APD at the TET end, and the baseband digital filtering process in TET DSP still remains; III) an electrical baseband filter is inserted between the APD and the ADC, and the baseband digital filtering process in the TET DSP is omitted; and IV) both the aforementioned electrical and optical filters are used, but the baseband digital filtering process in TET DSP is excluded.

Making use of digital filter lengths of $\mathrm{L}=64$ and drop RF signal parameters of $k=0.99$, three optical signals consisting of three OSOSW (OSTSW) 16-QAM signals and six OFDM subbands are generated to perform the drop operation. Utilizing above-mentioned four TET architectures to demodulate each dropped QAM and OFDM signal, the receiver sensitivity variations are plotted for various TET architectures in Fig. 7. The results indicate that the utilization of optical and baseband electrical analogue filters just alters the DPP by less

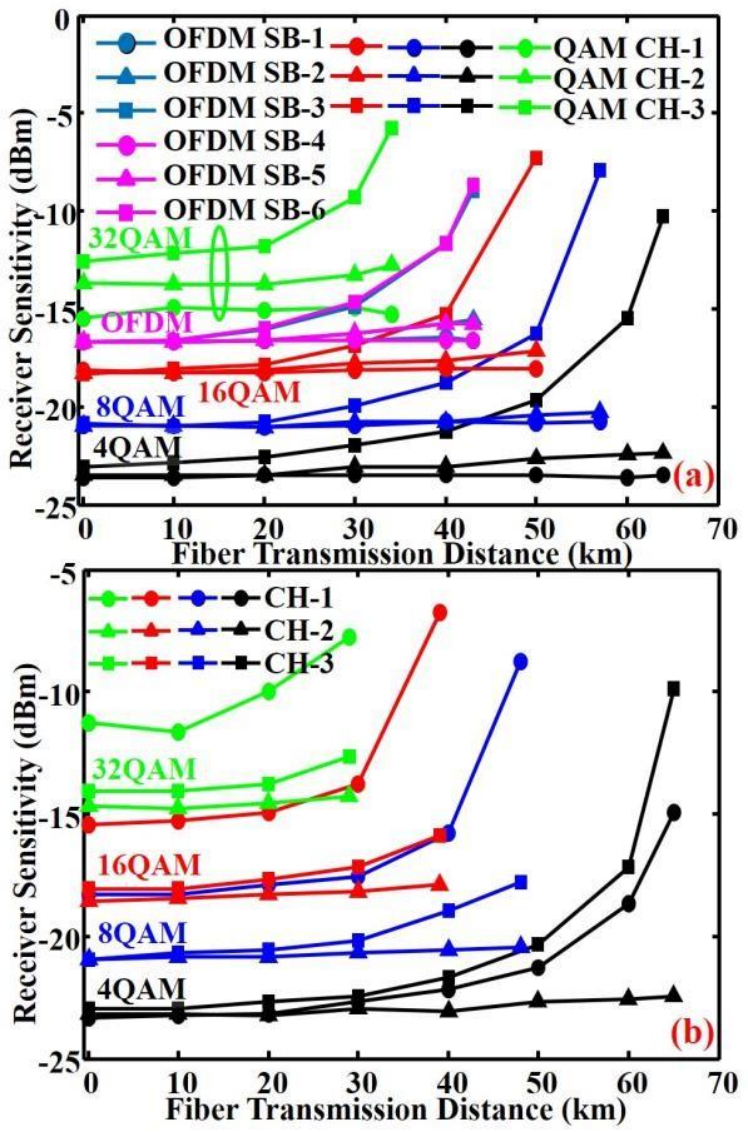

Fig. 8. ROADM drop performance robustness to fiber chromatic dispersion for different modulation formats; (a) OSOSW QAM and OFDM signals; (b) OSTSW QAM signals. CH: channel, SB: sub-band. than $1 \mathrm{~dB}$ for each OFDM and OSOSW/OSTSW QAM signals. This implies that the employment of optical and baseband electrical filters at the TET ends after the drop operation is unnecessary when employing digital filter lengths of $\mathrm{L} \geq 64$. This feature can simplify the terminal equipment configurations for costsensitive application scenarios such as optical network units (ONUs). It is also worth noting that the results presented in Sections III.A and III.B and results in

Section III.D are simulated using the Case I TET architectures. 


\section{Drop performance robustness to transmission system characteristics/impairments}

Given the fact that the ROADM add operation is very similar to the passive optical coupling-based combination of various optical upstream signals in the remote node of a PON network, and that the upstream signal transmission performances have been reported in [19], thus special attention in this subsection is mainly focused on the ROADM drop performance robustness to fiber chromatic dispersion.

Using digital filter lengths of $\mathrm{L}=64$, for the QAM and OFDM signals in the OSOSW/OSTSW schemes, the receiver sensitivity variations against different fiber transmission distance for different modulation formats are shown in Fig. 8, in obtaining which only the chromatic dispersion effect with a parameter of $16 \mathrm{ps} /(\mathrm{km} \cdot \mathrm{nm})$ is considered. After the fiber transmission, each of the QAM signals and OFDM sub-bands is dropped using a parameter $k$ of 0.99 . For simplicity, at the TET end, the LMS parameter set stated in Table II is still employed in recovering the received QAM signals. It can be seen in Fig. 8 that a low-order modulation format improves the ROADM drop performance robustness to fiber chromatic dispersion, and that compared with OSTSW QAM signals, the OSOSW QAM signals improve the ROADM drop performance robustness to fiber dispersion, because the balanced I/Q digital filtering maintains the signal recovery efficiency. It can also be seen in Fig. 8 that ROADMs can successfully drop both the OSOSW and OSTSW 16-QAM signals after transmitting over $40 \mathrm{~km}$ SSMF IMDD systems.

As the results in Fig. 8 are calculated using a fixed LMS parameter set optimized for back-to-back IMDD systems, the ROADM performance robustness, especially for the drop performance robustness can be further improved when more advanced adaptive signal recovery techniques are utilized, which may include modified decision-directed least mean square (DD-LMS) equalization [23] and hybrid adaptive equalization consisting of DD-LMS and modified CMMA aided pre-convergence [24].

\section{CONCLUSIONS}

Detailed numerical explorations have been undertaken of the ROADM performance robustness against variations in signal modulation formats, embedded DSP algorithms, transmission system impairments and terminal equipment configurations in IMDD-based optical network nodes. The numerical results have indicated that: 1) a long digital filter length improves the ROADM performance robustness, and when employing digital filter lengths larger than 64, the ROADMs have excellent performance robustness; 2) OFDM systems enable ROADMs to have the better performance robustness to digital filter length variations compared to QAM systems; 3) compared with OSTSW QAM scenarios, the OSOSW QAM systems enhance the ROADM performance robustness, and 4) for OSTSW QAM scenarios, the ROADM performance robustness can be further enhanced by narrowing the frequency intervals between frequency locations of I and Q components of the OSTSW QAM signals.

\section{ACKNOWLEDGMENT}

This work was supported in part by National Science Foundation of China No. 61571092, 61171045, Open Fund of State Key Laboratory at Shanghai Jiao Tong University No. 2013GZKF031301, Program for New Century Excellent Talents in University No. NCET-13-0099, Fundamental Research Funds for the Central Universities (No. ZYGX2013J005, ZYGX2013J001), Program for the 111

Project (B14039). This work was also supported in part by The Ser Cymru National Research Network in Advanced Engineering and Materials (NRN024 and NRN147), and in part by The Innovative UK TEROPON project.

\section{REFERENCES}

[1] T. L. Thanh, V. N. Q. Bao, P. T. Dat, A. Kanno, and T. Kawanishi, "10$\mathrm{Gb} / \mathrm{s}$ wireless signal transmission over a seamless IM/DD fiber-MMW system at $92.5 \mathrm{GHz}$," in IEEE Int. Conf. on Communications, London, U.K., 2015, pp. 1364-1369.

[2] C. Zhang, Q. Zhang, C. Chen, N. Jiang, D. Liu, K. Qiu, S. Liu, and B. Wu, "Metro-access integrated network based on optical OFDMA with dynamic sub-carrier allocation and power distribution," Opt. Express, vol. 21, no. 2, pp. 2474-2479, Jan. 2013.

[3] L. Valcarenghi, K. Kondepu, A. Sgambelluri, F. Cugini, P. Castoldi, G. R. de los Santos, R. A. Morenilla, and D. L. López, "Experimenting the integration of green optical access and metro networks based on SDN," in 17th Int. Conf. on Transparent Optical Networks, Budapest, 2015, paper We.D3.1.

[4] M. Ruffini, F. Slyne, C. Bluemm, N. Kitsuwan, and S. McGettrick, "Software defined networking for next generation converged metroaccess networks," Opt. Fiber Technol., vol. 26, pp. 31-41, Dec. 2015.

[5] B. Schrenk, A. Poppe, M. Stierle, and H. Leopold, "Fully-passive optical switch introducing dynamicity and flexibility to metro-access," IEEE Photon. Technol. Lett., vol. 27, no. 5, pp. 486-489, Feb. 2015.

[6] S. Gringeri, B. Basch, V. Shukla, R. Egorov, and T. J. Xia, "Flexible architectures for optical transport nodes and networks," IEEE Commun. Mag., vol. 48, no. 7, pp. 40-50, Jul. 2010.

[7] T. Watanabe, K. Suzuki, T. Goh, K. Hattori, A. Mori, T. Takahashi, T. Sakamoto, K. Morita, S. Sohma, and S. Kamei, "Compact PLC-based transponder aggregator for colorless and directionless ROADM," in OFC/NFOEC, Los Angeles, CA, USA, 2011, paper OTuD3.

[8] Z. Shen, H. Hasegawa, K. Sato, T. Tanaka, and A. Hirano, "A novel semiflexible grid optical path network that utilizes aligned frequency slot arrangement,"in ECOC, London, U.K., 2013, paper We.2.E.2.

[9] W. J. Jiang, A. Lebedev, Y. M. Lin, I. Vorobeichik, I. Gopp, A. Plotskiy, and W. I. Way, "Degree-expandable colorless, directionless, and contentionless ROADM without drop-side EDFAs," in OFC/NFOEC, Los Angeles, CA, USA, 2015, paper M3A.5.

[10] W. I. Way, "Optimum architecture for $\mathrm{M} \times \mathrm{N}$ multicast switch-based colorless, directionless, contentionless, and flexible-grid ROADM," in OFC/NFOEC, Los Angeles, CA, USA, 2012, paper NW3F.5.

[11] X. Yu, M. Tornatore, M. Xia, Y. Zhao, J. Zhang, and B. Mukherjee, "Brown-field migration from fixed grid to flexible grid in optical networks," in OFC/NFOEC, Los Angeles, CA, USA, 2015, paper W1I.4.

[12] S. Thiagarajan and S. Asselin, "Nodal contention in colorless, directionless ROADMs using traffic growth models," in OFC/NFOEC, Los Angeles, CA, USA, 2012, paper NW3F.2.

[13] M. D. Feuer, S. L. Woodward, P. Palacharla, X. Wang, I. Kim, and D. Bihon, "Intra-node contention in dynamic photonic networks," $J$. Lightwave Technol., vol. 29, no. 4, pp. 529-535, Feb. 2011.

[14] W. I. Way, P. N. Ji, and A. N. Patel, "Wavelength contention-free via optical bypass within a colorless and directionless ROADM [Invited]," $J$. Opt. Commun. Netw., vol. 5, no. 10, pp. A220-A229, Oct. 2013.

[15] A. Muhammad, G. Zervas, G. Saridis, E. H. Salas, D. Simeonidou, and R. Forchheimer, "Flexible and synthetic SDM networks with multi-corefibers implemented by programmable ROADMs," in ECOC, Cannes, France, 2014, paper P.6.6. 
[16] W. Jin, X. Duan, M. Bolea, R. P. Giddings, N. Jing, C. F. Zhang, K. Qiu, and J. M. Tang, "New ROADMs with DSP-enabled dynamic and flexible operations for elastic optical networks," in OFC/NFOEC, Los Angeles, CA, USA, 2015, paper Th2A.50.

[17] W. Jin, X. Duan, Y. Dong, B. Cao, R. P. Giddings, C. F. Zhang, K. Qiu, and J. M. Tang, "DSP-enabled flexible ROADMs without optical filters and O-E-O conversions," J. Lightwave Technol., vol. 33, no. 19, pp. 41244131, Oct. 2015

[18] X. Duan, M. L. Deng, W. Jin, R. P. Giddings, S. Mansoor, and J. M. Tang, "Experimental demonstration of DSP-enabled drop operations of flexible ROADMs excluding optical filters and O-E-O conversions," in $O F C$, Anaheim, CA, USA, 2016, paper M3E.4.

[19] M. Bolea, R. P. Giddings, M. Bouich, C. Aupetit-Berthelemot, and J. M. Tang, "Digital filter multiple access PONs with DSP-enabled software reconfigurability," J. Opt. Commum. Netw., vol. 7, no. 4, pp. 215-222, Apr. 2015.

[20] X. Duan, R. P. Giddings, M. Bolea, Y. Ling, B. Cao, S. Mansoor, and J. M. Tang, "Real-time experimental demonstrations of software reconfigurable optical OFDM transceivers utilizing DSP-based digital orthogonal filters for SDN PONs," Opt. Express, vol. 22, no. 16, pp. 19674-19685, Aug. 2014.

[21] M. Bolea, R. P. Giddings, and J. M. Tang, "Digital orthogonal filter enabled optical OFDM channel multiplexing for software-reconfigurable elastic PONs," J. Lightwave Technol., vol. 32, no. 6, pp. 1200-1206, Mar. 2014.

[22] J. Yang and G. E. Sobelman, "Sparse LMS with segment zero attractors for adaptive estimation of sparse signals," in IEEE Asia Pacific Conf. on Circuits and Systems, Kuala Lumpur, 2010, pp. 422-425.

[23] J. Zhang, X. Li, Y. Xia, Y. Chen, J. Yu, X. Chen, and J. Xiao, "60-Gb/s CAP-64QAM transmission using DML with direct detection and digital equalization," in $O F C / N F O E C$, San Francisco, CA, USA, 2014, paper W1F.3.

[24] L. Tao, Y. Wang, Y. Gao, A. P. T. Lau, N. Chi, and C. Lu, "40 Gb/s CAP32 system with DD-LMS equalizer for short reach optical transmissions," IEEE Photon. Technol. Lett., vol. 25, no. 23, pp. 2346-2349, Dec. 2013. 